\title{
The magnetic field and the location of the TeV emitter in Cygnus X-1 and LS 5039
}

\author{
V. Bosch-Ramon ${ }^{1}$, D. Khangulyan ${ }^{1}$, and F. A. Aharonian ${ }^{2,1}$ \\ 1 Max Planck Institut für Kernphysik, Saupfercheckweg 1, Heidelberg 69117, Germany \\ e-mail: [vbosch;Dmitry.Khangulyan] @mpi-hd .mpg.de \\ 2 Dublin Institute for Advanced Studies, Dublin, Ireland \\ e-mail: Felix.Aharonian@dias.ie
}

Received 21 July 2008 / Accepted 15 August 2008

ABSTRACT

\begin{abstract}
Context. Cygnus X-1 and LS 5039 are two X-ray binaries observed at TeV energies. Both sources are compact systems, contain jet-like (radio) structures, and harbor very luminous $\mathrm{O}$ stars. A $\mathrm{TeV}$ signal has been found around the superior conjunction of the compact object in both objects, when the highest gamma-ray opacities are expected.

Aims. We investigate the implications of finding TeV emission from Cygnus X-1 and LS 5039 around the superior conjunction, since this can give information on the system magnetic field and the location of the $\mathrm{TeV}$ emitter.

Methods. Using the very high-energy spectra and fluxes observed around the superior conjunction in Cygnus X-1 and LS 5039, we compute the absorbed luminosity that is caused by pair creation in the stellar photon field for different emitter positions with respect to the star and the observer line of sight. The role of the magnetic field and electromagnetic cascading are discussed. For the case of inefficient electromagnetic cascading, the expected secondary synchrotron fluxes are compared with the observed ones at X-ray energies.

Results. We find that, in Cygnus X-1 and LS 5039, either the magnetic field in the star surroundings is much smaller than the one expected for $\mathrm{O}$ stars or the $\mathrm{TeV}$ emitter is located at a distance $>10^{12} \mathrm{~cm}$ from the compact object.

Conclusions. Our results strongly suggest that the TeV emitters in Cygnus X-1 and LS 5039 are located at the borders of the binary system and well above the orbital plane. This would not agree with those models for which the emitter is well inside the system, like the innermost-jet region (Cygnus X-1 and LS 5039; microquasar scenario) or the region between the pulsar and the primary star (LS 5039; standard pulsar scenario).
\end{abstract}

Key words. gamma rays: theory - X-rays: binaries - radiation mechanisms: non-thermal

\section{Introduction}

Cygnus X-1 and LS 5039 are high-mass X-ray binaries presenting radiation in the very high-energy (VHE) range (Aharonian et al. 2005; Albert et al. 2007). Both sources show extended outflows that generate non-thermal radio emission (Stirling et al. 2001; Paredes et al. 2000) and are located at distances of $\approx 2.1 \mathrm{kpc}$ (Ziolkowski 2005) and $\approx 2.5 \mathrm{kpc}$ (Casares et al. 2005; C05 hereafter), respectively. The primary object in these two systems is an O star (Gies \& Bolton 1986; C05), and the compact object is a $\sim 20 M_{\odot}$ black hole in the case of Cygnus X-1 (Ziolkowski 2005) and is still of unknown nature in the case of LS 5039 (C05). The detection of jet-like radio emitting structures has led to the classification of these sources as microquasars (Stirling et al. 2001; Paredes et al. 2000). Later on, however, not detecting accretion features in the X-ray spectrum of LS 5039 has been interpreted as a hint of a non-accreting pulsar (e.g. Martocchia et al. 2005). On the other hand, Cygnus X-1 is a firmly established accreting source (e.g. Pringle \& Rees 1972). It is worth noting that LS 5039 has been associated to an EGRET source (Paredes et al. 2000), whereas Cygnus X-1 has no GeV association.

Cygnus X-1 and LS 5039 are relatively similar to each other, as shown in Table 1 . Besides showing extended radio emission, the two systems harbor massive and hot stars, have quite compact size, $\sim 0.1-0.2 \mathrm{AU}$, and have shown a TeV signal around the superior conjunction of the compact object (SUPC). The TeV emission properties of both sources around SUPC are presented as well in Table 1 (bottom). In the case of Cygnus X-1, evidence of detection above $100 \mathrm{GeV}$ of $4.1 \sigma$ significance has been reported once right before SUPC, at phase $\approx 0.9$ (Albert et al. 2007). In LS 5039, the VHE emission has been clearly detected $(40 \sigma)$ all along the orbit, being in fact periodic (Aharonian et al. 2006a) with the orbital period (C05). At the phase $0.00 \pm 0.05(\sim$ SUPC $)$, the detection significance is $6.1 \sigma$. There is however a big difference between the two sources. Whereas Cygnus X-1 shows a thermal (comptonized) hard X-ray spectrum (e.g. Sunyaev \& Trumper 1979), with a luminosity $L_{\mathrm{X}} \sim 10^{37} \mathrm{erg} \mathrm{s}^{-1}$ in the epoch when the TeV emission was detected (Albert et al. 2007), the X-ray radiation from LS 5039 is three orders of magnitude less luminous, i.e. $L_{\mathrm{X}} \sim$ $10^{34} \mathrm{erg} \mathrm{s}^{-1}$, and could be dominated by a non-thermal component (e.g. Bosch-Ramon et al. 2007).

In Cygnus X-1 and LS 5039, if the TeV emitter is deep inside the system and behind the primary star around SUPC, the photon-photon absorption opacity $(\tau)$ for $\mathrm{TeV}$ gamma rays in the stellar photon field will be $\gg 1$ in the direction of the observer. In this situation, either the magnetic field is low enough to allow efficient electromagnetic (EM) cascading to develop (e.g. Aharonian et al. 2006b; Bednarek \& Giovanelli 2005; 
Table 1. Properties of the binary systems Cygnus X-1 and LS 5039.

\begin{tabular}{|c|c|c|}
\hline & Cygnus X-1 & LS 5039 \\
\hline Star luminosity [erg s ${ }^{-1}$ ] & $1.3 \times 10^{39^{b}}$ & $7 \times 10^{38^{a}}$ \\
\hline Star temperature $[\mathrm{K}]$ & $3 \times 10^{4^{b}}$ & $3.8 \times 10^{4^{a}}$ \\
\hline Stellar radius $[\mathrm{cm}]$ & $1.5 \times 10^{12^{b}}$ & $7 \times 10^{11^{a}}$ \\
\hline Orbital semi-major axis $[\mathrm{cm}]$ & $3.5 \times 10^{12^{b}}$ & $2.1 \times 10^{12^{a}}$ \\
\hline Orbital distance at SUPC $\left[R_{*}\right]$ & $\approx 2.3$ & $\approx 2$ \\
\hline Distance $[\mathrm{kpc}]$ & $2.1^{b}$ & $2.5^{a}$ \\
\hline Eccentricity & $0^{c}$ & $0.35^{a}$ \\
\hline Inclination & $30^{\circ d}$ & $\sim 20^{\circ}-60^{\circ}$ \\
\hline Wind velocity $\left[\mathrm{cm} \mathrm{s}^{-1}\right]$ & $\approx 2 \times 10^{8^{e}}$ & $\approx 2 \times 10^{8^{a}}$ \\
\hline Mass loss rate $\left[M_{\odot} \mathrm{yr}^{-1}\right]$ & $\approx 2 \times 10^{-6^{f}}$ & $\approx 5 \times 10^{-7 a}$ \\
\hline Luminosity [>100 GeV]; $\left.\mathrm{erg} \mathrm{s}^{-1}\right]$ & $\approx 1.6 \times 10^{34^{9}}$ & $\approx 4 \times 10^{33^{h}}$ \\
\hline Spectral index & $3.2 \pm 0.6^{g}$ & $2.6 \pm 0.3^{h}$ \\
\hline
\end{tabular}

${ }^{a}$ C05; ${ }^{b}$ Ziolkowski (2005); ${ }^{c}$ Gies \& Bolton $(1982){ }^{d}$ Gies \& Bolton (1986); ${ }^{e}$ Herrero et al. (1995); ${ }^{f}$ Gies et al. (2003); ${ }^{g}$ Albert et al. (2007); ${ }^{h}$ Aharonian et al. (2006a).

Orellana et al. 2007), effectively reducing $\tau$ around SUPC, or the absorbed energy will be reradiated mainly by secondary pairs via synchrotron emission (e.g. Bosch-Ramon et al. 2008). In the latter case, the high (effective) opacities will require an injected gamma-ray luminosity much higher than the observed one, and the synchrotron luminosity of the secondary pairs will be similar to that of the absorbed gamma rays. Because the secondary synchrotron luminosity should not exceed the observational constraints, we can put strong restrictions on either the magnetic field strength or on the emitter location with respect to the star and the line of sight of the observer. In this work, we discuss the implications of two possible situations, i.e. a low versus a high magnetic field in the stellar surroundings for the $\mathrm{TeV}$ emitters in Cygnus X-1 and LS 5039.

\section{The magnetic field in the stellar surroundings}

If the $\mathrm{TeV}$ emitter moves along the orbit close to the compact object, the maximum in photon-photon absorption will take place around SUPC (e.g. see Fig. 14 in Khangulyan et al. 2008 -K08 hereafter). For sources like Cygnus X-1 and LS 5039, $\tau$ is $\approx 20$ for $i=45^{\circ}$ and $300 \mathrm{GeV}$ photons emitted in the vicinity of the compact object, around SUPC. This shows that approximately $10^{9}$ times more radiation should be produced to obtain the observed gamma-ray fluxes. This estimate gives an idea of the amount of energy that can be required to power the observed VHE radiation.

The required energy budget to power the VHE emission around SUPC can be significantly reduced through efficient EM cascading, which occurs if IC scattering takes place deep enough in the Klein Nishina $(\mathrm{KN})$ regime, as is the case for electrons of energy $\gtrsim \mathrm{TeV}$ in the stellar photon field. Nevertheless, the dominance of KN IC energy losses for TeV electrons requires a magnetic field strength well below a critical value, $B_{\mathrm{c}}$, since otherwise a substantial fraction of the energy will be radiated via the synchrotron process. The value of $B_{\mathrm{c}}$ for $\mathrm{TeV}$ electrons is defined by the balance of synchrotron and IC energy losses (K08):

$B_{\mathrm{c}} \approx 10\left(\frac{L_{*}}{10^{39} \mathrm{erg} \mathrm{s}^{-1}}\right)^{1 / 2}\left(\frac{R_{*}}{R}\right) \mathrm{G}$,

where $R, R_{*}$, and $L_{*}$ are the star distance, radius, and luminosity, respectively.
To know whether efficient EM cascading can occur requires the magnetic field strength expected in the surroundings of the $\mathrm{TeV}$ emitter, $B_{*}$, at $1-2 R_{*}$ from the O-star surface. The magnetic fields close to the stellar surface has been only directly measured in several O stars $\left(\theta^{1}\right.$ Ori C, Donati et al. 2006; HD 191612, Wade et al. 2006; and $\zeta$ Orionis A, Bouret et al. 2008, for clear detections; and HD 36879, HD 148937, HD 152408, and HD 164794, Hubrig et al. 2008 for $3-4 \sigma$ detections) due to the difficulties detecting the Zeeman effect, finding however relatively high values of $\sim 100-1000 \mathrm{G}$. In addition, it has been speculated that magnetic fields could be a common feature of massive stars, as inferred from X-ray photometric and spectroscopic results (e.g. Stelzer et al. 2005; Waldron \& Cassinelli 2007), non thermal radio synchrotron emission (e.g. Benaglia 2005; Schnerr et al. 2007), cyclical variations in UV wind spectral lines (e.g. Fullerton 2003; Kaper et al. 1996), or magnetic fields in neutron stars assuming a fossil origin (e.g. Ferrario \& Wickramasinghe 2006). Finally, the $R$-dependence of $B_{*}$ could be, roughly, $\propto 1 / R^{1,2,3}$ depending on $R$ (i.e. a toroidal, radial, or poloidal dominant component; Usov \& Melrose 1992). Even in the extreme case of $B_{*} \propto 1 / R^{3}$ and $100 \mathrm{G}$ at the stellar surface, $B_{*} \gtrsim B_{\mathrm{c}}$ at $R \sim 2 R_{*}$, which would prevent efficient EM cascading (although $B_{*} \ll B_{\text {c }}$ still cannot be discarded).

\section{Location of the emitter}

As seen in Sect. 2, under $B_{*}>B_{\text {c }}$ most of the absorbed energy is reemitted via synchrotron emission. The corresponding characteristic frequency and the short radiation timescales of secondary pairs with energies around or a bit larger than the pair creation threshold $\left(\sim m_{\mathrm{e}}^{2} c^{4} / 3 k T_{*}\right), 100-1000 \mathrm{GeV}$, determine that the synchrotron emission will peak at X-rays or will flatten in the X-ray to gamma-ray range (K08; Bosch-Ramon et al. 2008). This cannot be prevented by particle escape, since the radiative timescales at the relevant particle energies will be shorter than the escape ones even when particles move at $c$. That this X-ray secondary synchrotron emission cannot overcome the observed $\mathrm{X}$-ray fluxes restricts the location of the $\mathrm{TeV}$ emitter.

To estimate the amount of energy that could be released via synchrotron emission in X-rays, we plotted 2-dimensional maps of the absorbed luminosity depending on the location of the emitter in the binary system. These maps are presented in Figs. 1 and 2 for Cygnus X-1 and LS 5039, respectively. The XY coordinates correspond to the plane formed by the emitter and star positions and by the observer line of sight. To compute these maps, we deabsorbed the observed spectra and fluxes $\gtrsim 100 \mathrm{GeV}$ around SUPC in both Cygnus X-1 and LS 5039 (see Table 1). The regions forbidden by the X-ray observational constraints in Cygnus X-1 and LS 5039 are limited by a contour line. We computed the 2-dimensional maps for both sources assuming an anisotropic (e.g. stellar photon IC) and an isotropic mechanism for the production of primary gamma rays. Both cases gave quite similar results, thus we chose the most conservative one for each source, i.e. an anisotropic mechanism in the case of Cygnus X-1 and an isotropic one in the case of LS 5039. We also explored the impact of adopting the observational lower- and upper-limits for the spectral slopes (see Table 1), obtaining only small differences between the resulting maps. Therefore, we adopted the mean observed spectral indexes to create the plots presented in Figs. 1 and 2.

The computed maps are $i$-independent, since they show the amount of absorbed luminosity for different emitter locations in the emitter-star-observer plane, wherever the compact object is located. However, for illustrative purposes, we show the position 


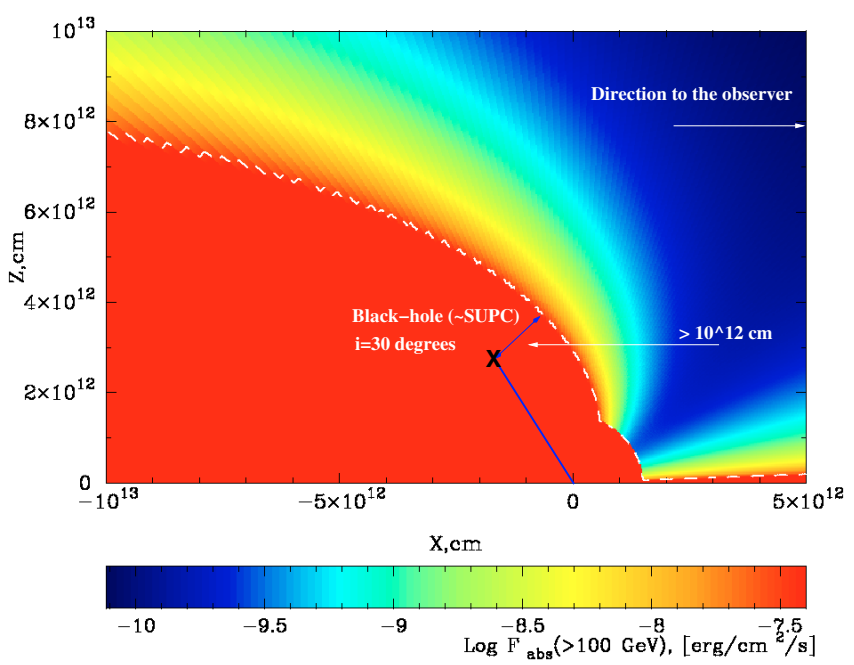

Fig. 1. Two-dimensional map for Cygnus $X-1$ of the amount of flux absorbed via photon-photon interactions for different $\mathrm{TeV}$ emitter locations within the system. The $X Y$-plane is the one that joins the observer, the star, and the emitter, and the $X$-direction is the one joining the star and the observer (to the right). The $\mathbf{X}$ in the plot represents the location of the compact object at SUPC for an inclination angle of $30^{\circ}$. The region to the left of the long-dashed line is forbidden by the constraints from X-ray observations as a location of the $\mathrm{TeV}$ emitter for $B_{*}$ well above $B_{\mathrm{c}}$. The emitter has to be at a distance $>10^{12} \mathrm{~cm}$ between the compact object and the closer point in the X-ray flux limit curve.

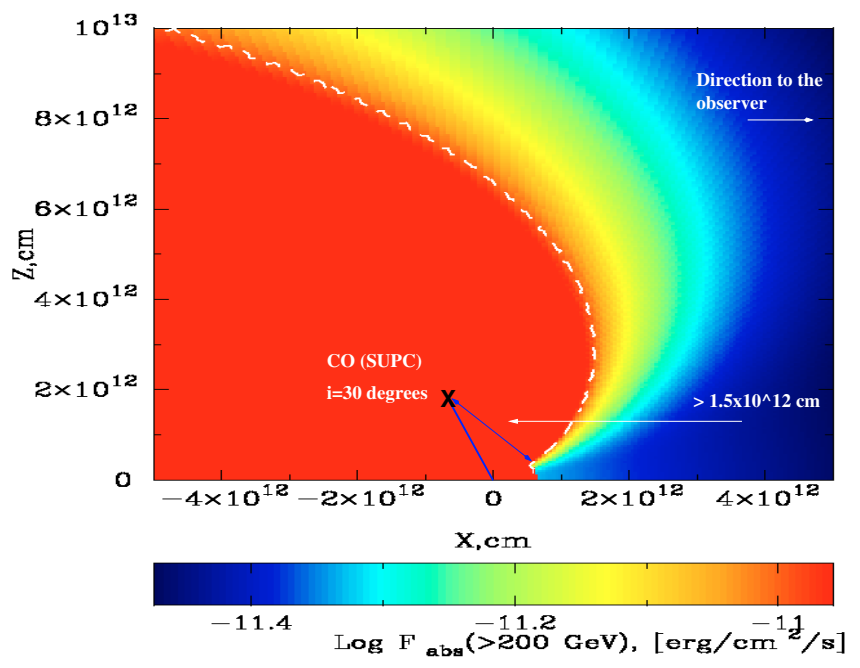

Fig. 2. The same as in Fig. 1 but for LS 5039. In this case, the emitter location is constrained to distances over a few $10^{12} \mathrm{~cm}$.

of the compact object in the emitter-star-observer plane when $i=30^{\circ}$. This plus the contour lines give an idea of where the emitter could be with respect to the compact object. As seen in Figs. 1 and 2 , the TeV emitter can hardly be closer than $\approx 10^{12} \mathrm{~cm}$ from the black hole in Cygnus X-1. For the case of LS 5039, the minimum distance is a bit larger. It is remarkable that the energy requirements for an emitter close to the compact object grow for larger $i$ (i.e. towards a neutron star mass for the compact object C05-), since larger inclinations locate the compact object deeper in the forbidden regions (see also Fig. 6 in Böttcher 2007). We also note that, since $\tau \propto 1 / R$ (e.g. K08), the energy requirements would even be stronger if the TeV emitter were located in the region between the compact object and the star.

In Fig. 3, we show the spectral energy distribution (SED) of the synchrotron radiation from the secondary pairs in LS 5039

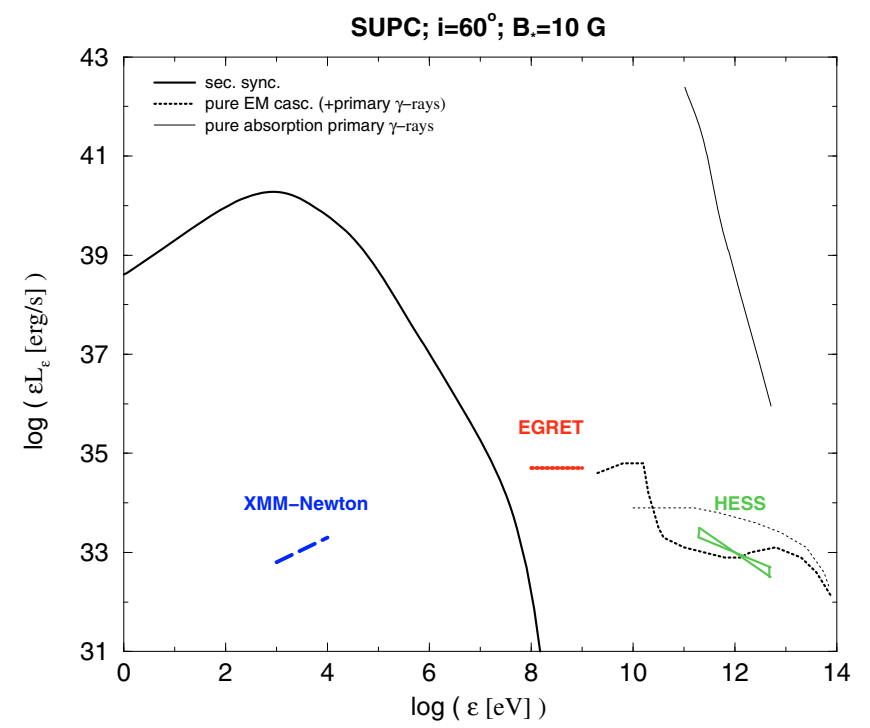

Fig. 3. The SED of the synchrotron emission (solid line) produced by the secondary pairs (derived from the deabsorbed primary gamma-ray spectrum) and the SED for pure EM cascading (dotted line) are presented for LS 5039. The TeV emitter is located close to the compact object at SUPC, and the inclination angle is $i=60^{\circ}$ and, for the secondary emission case, $B_{*}=10 \mathrm{G}$. The absorbed luminosity in the secondary case is about $\sim 10^{42} \mathrm{erg} \mathrm{s}^{-1}$. For the pure cascade case, the injected luminosity in primary gamma rays is $\sim 3 \times 10^{35} \mathrm{erg} \mathrm{s}^{-1}$. The SEDs observed by XMM-Newton (X-rays) close to SUPC (at phase 0.0; Bosch-Ramon et al. 2007), EGRET (GeV) (time averaged; Hartman et al. 1999), and HESS (phase interval: 0.0-0.1; Aharonian et al. 2006a), are also shown.

at SUPC, assuming that the $\mathrm{TeV}$ emitter is in the vicinity of the compact object. The deabsorbed and the primary gamma-ray spectra are presented as well. We took $i=60^{\circ}$, which would correspond to the non-accreting pulsar scenario, and $B_{*}=10 \mathrm{G}^{1}$. In the same plot, for illustrative purposes, a computed pure IC cascade SED $\left(B_{*} \ll B_{\mathrm{c}}\right)$, roughly similar to the observed VHE SED at SUPC, is shown. As can be seen in the figure, pure absorption and secondary emission renders very large synchrotron X-ray fluxes, far above the observed ones. Otherwise, the occurrence of pure EM cascading can effectively reduce $\tau$ by several orders of magnitude, and it yields as well GeV luminosities consistent with the EGRET ones (see also Aharonian et al. 2006b). In the case of Cygnus X-1, the secondary synchrotron SED, not shown here, would look similar to LS 5039, although the observed X-ray fluxes would appear $\sim 4$ orders of magnitude below the computed X-ray fluxes, instead of the $\sim 8$ of LS 5039.

We emphasize that the precise shape of the observed spectra, which is difficult to obtain from the low statistics of the data around SUPC, is not important for our calculations, provided that the deabsorbed spectrum is very soft, as seen in Fig. 3 for LS 5039. In the case of Cygnus X-1 (not shown here), the deabsorbed SED is roughly similar to the one of LS 5039.

\section{Discussion}

The detection of Cygnus X-1 and LS 5039 around SUPC, plus $\mathrm{X}$-ray data, give strong constraints on the magnetic field and the location of the $\mathrm{TeV}$ emitter in these systems. In summary, if the $\mathrm{TeV}$ emitter is close to the compact object, the magnetic field in the stellar surroundings must be smaller than a few Gauss. These

\footnotetext{
1 The magnetic field can be taken as homogeneous in the secondary synchrotron emitter, since the large $\tau$ implies that most of the radiation originates in a small region, of size $\ll R$.
} 
values will allow IC cascades to develop, effectively reducing the photon-photon absorption opacities. However, this magnetic field seems rather low when compared with the values expected in $\mathrm{O}$ stars. For a more realistic $B_{*}>B_{\mathrm{c}}$, EM cascading becomes inefficient, and the large amount of energy absorbed in the stellar photon field will be reemitted by the secondary pairs mainly via the synchrotron process. For $B_{*}>B_{\mathrm{c}}$, this radiation will peak roughly in the X-ray band. In such a case, to avoid the violation of the observational constraints, the TeV emitter must be located at distances $>10^{12} \mathrm{~cm}$ from the compact object in both Cygnus X-1 and LS 5039. It is consistent with how, as noted by $\mathrm{K} 08$, extreme acceleration rates are required to explain the highest energy photons from LS 5039 if the accelerator is deep inside the system. Remarkably, the low hydrogen column densities inferred using X-ray data would also hint at an X-ray emitter far from the compact object (Bosch-Ramon et al. 2007).

A $\mathrm{TeV}$ emitter far from the compact object requires a physical mechanism to transport energy to the borders of the system to power the VHE emission. The source of this energy could be accretion in Cygnus X-1, or either accretion (microquasar scenario) or a powerful pulsar wind (non-accreting pulsar scenario) in LS 5039. The energy carrier could be the jet seen in radio in Cygnus X-1. In the case of LS 5039, energy could be transported by a jet (the jet-like structure seen in radio) powered by radiatively inefficient accretion (e.g. Bogovalov \& Kelner 2005), since no accretion X-ray features have been found. In the standard pulsar scenario for LS 5039 (e.g. Dubus 2008; SierpowskaBartosik \& Torres 2008), the TeV emitter would be located close to the pulsar or around the line joining the pulsar and the star. This would imply that, for reasonable energy budgets, very litthe emission should be expected due to absorption around SUPC (see Fig. 2 in Dubus 2006 - lower panel; Fig. 4 in Dubus et al. 2008; and Fig. 2 in Sierpowska-Bartosik \& Torres 2008), unlike how it is observed. This does not agree with the standard pulsar scenario for LS 5039, although in a more general case, a supersonic outflow produced in the star/pulsar wind colliding region (see Bogovalov et al. 2008) may transport energy efficiently to the system borders and farther out.

To conclude, our results strongly suggest that the TeV emitters in Cygnus X-1 and LS 5039 are located at the borders of the binary system, well above the orbital plane. This would not be compatible with those models for which the emitter is well inside the system, like the innermost-jet region (Cygnus X-1 and LS 5039; microquasar scenario) or the region between the pulsar and the primary star (LS 5039; standard pulsar scenario).

Acknowledgements. The authors thank the anonymous referee for constructive comments. V.B-R. gratefully acknowledges support from the Alexander von Humboldt Foundation. V.B-R. acknowledges support by D.G.I. of M.E.C. under grant AYA2007-68034-C03-01, as well as partial support by the European Regional Development Fund (ERDF/FEDER).

\section{References}

Albert, J., Aliu, E., Anderhub, H., et al. 2007, ApJ, 665, L51

Aharonian, F., Akhperjanian, A. G., Aye, K. M., et al. 2005, Science, 309, 746 Aharonian, F., Akhperjanian, A. G., Bazer-Bachi, A. R., et al. 2006a, A\&A, 460, 743

Aharonian, F. A., Anchordoqui, L. A., Khangulyan, D., \& Montaruli, T. 2006b, J. Phys. Conf. Ser., 39, 408

Bednarek, W., \& Giovannelli, F. 2007, A\&A, 464, 437

Benaglia, P. 2005, BAAA, 48, 127

Bogovalov, S. V., \& Kelner, S. R. 2005, Astron. Rep., 49, 57

Bogovalov, S. V., Khangulyan, D., Koldoba, A. V., Ustyugova, G. V., \& Aharonian, F. 2008, MNRAS, 387, 63

Bosch-Ramon, V., Motch, C., \& Ribó, M., et al. 2007, A\&A, 473, 545

Bosch-Ramon, V., Khangulyan D., \& Aharonian, F. 2008, A\&A, 482, 397

Böttcher, M. 2007, APh, 27, 278

Bouret J. C., Donati J. F., \& Martins F. 2008 [arXiv: 0806.2162]

Casares, J., Ribó, M., Ribas, I., et al. 2005, MNRAS, 364, 899 (C05)

Donati, J.-F., Howarth, I. D., Bouret, J.-C., et al. 2006, MNRAS, 365, L6

Dubus, G. 2006, A\&A, 451, 9

Dubus, G. 2008, A\&A, 477, 691

Ferrario, L., \& Wickramasinghe, D. 2006, MNRAS, 367, 1323

Fullerton, A. W. 2003, in Magnetic Fields in O, B and A Stars, ed. L. A. Balona,

H. F. Henrichs, R. Medupe, ASPC Ser. 305, 3335

Gies, D. R., \& Bolton, C. T. 1982, ApJ, 260, 240

Gies, D. R., \& Bolton, C. T. 1986, ApJ, 304, 371

Gies, D. R., Bolton, C. T., \& Thomson, J. R. 2003, ApJ, 583, 424

Hartman, R. C., Bertsch, D. L., \& Bloom, S. D. et al. 1999, ApJS, 123, 79

Herrero, A., Kudritzki, R. P., Gabler, R., Vilchez, J. M., \& Gabler, A. 1995, A\&A, 297, 556

Hubrig, S., Schoeller, M., Schnerr, R. S., et al. 2008, A\&A,

in press [arXiv:0808.2039]

Kaper, L., Henrichs, H. F., Nichols, J. S., et al. 1996, A\&AS, 116, 257

Khangulyan, D., Aharonian, F., \& Bosch-Ramon, V. 2008, MNRAS, 383, 467 (K08)

Martocchia, A., Motch, C., \& Negueruela, I. 2005, A\&A, 430, 245

Orellana, M., Bordas, P., Bosch-Ramon, V., Romero, G. E., \& Paredes, J. M. 2007, A\&A, 476, 9

Paredes, J. M., Martí, J., Ribó, M., \& Massi, M. 2000, Science, 288, 2340

Pringle, J. E., \& Rees, M. J. 1972, A\&A, 21, 1

Russell, D. M., Fender, R. P., Gallo, E., \& Kaiser, C. R. 2007, MNRAS, 376, 1341

Schnerr, R. S., Rygl, K. L. J., \& van der Horst, A. J. 2007, A\&A, 470, 1105

Sierpowska-Bartosik, A., \& Torres, D. F. 2008, ApJ, 674, 89

Stelzer, B., Flaccomio, E., \& Montmerle, T. 2005, ApJS, 160, 557

Stirling, A. M., Spencer, R. E., \& de la Force, C. J. 2001, MNRAS, 327, 1273

Sunyaev, R. A., \& Truemper, J. 1979, Nature, 279, 506

Usov, V. V., \& Melrose, D. B. 1992, ApJ, 395, 575

Wade, G. A., Fullerton, A. W., Donati, J.-F., et al. 2006, A\&A, 451, 195

Waldron, W. L., \& Cassinelli, J. P. 2007, ApJ, 668, 456

Ziolkowski, J. 2005, MNRAS, 358, 851 